MATHEMATICS OF COMPUTATION

Volume 74, Number 250, Pages 841-852

S 0025-5718(04)01686-2

Article electronically published on May 28, 2004

\title{
AN INFINITE FAMILY OF BOUNDS ON ZEROS OF ANALYTIC FUNCTIONS AND RELATIONSHIP TO SMALE'S BOUND
}

\author{
BAHMAN KALANTARI
}

\begin{abstract}
Smale's analysis of Newton's iteration function induce a lower bound on the gap between two distinct zeros of a given complex-valued analytic function $f(z)$. In this paper we make use of a fundamental family of iteration functions $B_{m}(z), m \geq 2$, to derive an infinite family of lower bounds on the above gap. However, even for $m=2$, where $B_{2}(z)$ coincides with Newton's, our lower bound is more than twice as good as Smale's bound or its improved version given by Blum, Cucker, Shub, and Smale. When $f(z)$ is a complex polynomial of degree $n$, for small $m$ the corresponding bound is computable in $O(n \log n)$ arithmetic operations. For quadratic polynomials, as $m$ increases the lower bounds converge to the actual gap. We show how to use these bounds to compute lower bounds on the distance between an arbitrary point and the nearest root of $f(z)$. In particular, using the latter result, we show that, given a complex polynomial $f(z)=a_{n} z^{n}+\cdots+a_{0}, a_{n} a_{0} \neq 0$, for each $m \geq 2$ we can compute upper and lower bounds $U_{m}$ and $L_{m}$ such that the roots of $f(z)$ lie in the annulus $\left\{z: L_{m} \leq|z| \leq U_{m}\right\}$. In particular, $L_{2}=$ $\frac{1}{2} / \max \left\{\left|a_{k} / a_{0}\right|^{1 / k}: k=1, \ldots, n\right\}, U_{2}=2 \max \left\{\left|a_{n-k} / a_{n}\right|^{1 / k}: k=1, \ldots, n\right\}$; and $L_{3}=[(\sqrt{5}-1) / 2] / \max \left\{\left(\left|a_{1} a_{k-1}-a_{0} a_{k}\right| /\left|a_{0}^{2}\right|\right)^{1 / k}: k=2, \ldots, n+1\right\}$, $U_{3}=[(\sqrt{5}+1) / 2] \max \left\{\left(\left|a_{n-1} a_{n-k+1}-a_{n} a_{n-k}\right| /\left|a_{n}^{2}\right|\right)^{1 / k}: k=2, \ldots, n+\right.$ $1\}$, where $a_{-1}=a_{n+1}=0$. An application of the latter bounds is within Weyl's classical quad-tree algorithm for computing all roots of a given complex polynomial.
\end{abstract}

\section{INTRODUCTION}

Let $f(z)$ be a complex-valued function analytic everywhere on the complex plane. For a complex number $c=a+i b$, its modulus is denoted by $|c|=\sqrt{a^{2}+b^{2}}$. Consider Newton's iteration function

$$
N(z)=z-\frac{f(z)}{f^{\prime}(z)}
$$

Define

$$
\gamma(z)=\sup \left\{\left|\frac{f^{(k)}(z)}{f^{\prime}(z) k !}\right|^{1 /(k-1)}, \quad k \geq 2\right\}
$$

Received by the editor May 5, 2003 and, in revised form, October 2, 2003.

2000 Mathematics Subject Classification. Primary 12D10, 65H05, 30C15, 68Q25.

Key words and phrases. Newton's method, Smale's separation lower bound, fixed-points, basic family.

(C)2004 American Mathematical Society 
From Smale's analysis of the one-point theory for Newton's method the following theorem is deducible:

Theorem 1.1 (Smale 23]). If $\xi, \xi^{\prime}$ are distinct zeros of $f, \xi$ a simple zero, then they are separated by a distance

$$
\left|\xi-\xi^{\prime}\right| \geq \frac{3-\sqrt{7}}{2 \gamma(\xi)} \approx \frac{.177}{\gamma(\xi)} .
$$

The following stronger lower bound is given in Blum et al. 1] (Corollary 1, page 158):

$$
\left|\xi-\xi^{\prime}\right| \geq \frac{5-\sqrt{17}}{4 \gamma(\xi)} \approx \frac{.219}{\gamma(\xi)}
$$

Such theorems are referred as separation theorems. Dediue 2] gives separation theorems for systems of complex polynomials and in particular polynomials in one complex variable.

In this paper we will derive a family of lower bounds indexed by an integer $m \geq 2$ on the gap of Theorem 1.1, which in particular when $m=2$ improves (1.3) as well as (1.4) by replacing their lower bounds with $1 /(2 \gamma(\xi))$, which is more than twice as good. Our results make use of a fundamental family of iteration functions, called the basic family, $\left\{B_{m}(z), m=2, \ldots\right\}$. The order of convergence of each $B_{m}(z)$ to a simple root of $f(z)$ is $m$. Moreover, $B_{2}(z)$ coincides with Newton's iteration function.

The paper is organized as follows: In Section 2, we describe the basic family and its significant relevant properties for complex polynomials. We then extend these to the case of analytic functions. In Section 3, we make use of the basic family to derive lower bounds on the distance from a simple zero of $f$ to its nearest distinct zero. In Section 4, we make use of the preceding lower bounds to obtain lower bounds on the distance between an arbitrary point and the nearest root of $f$. In particular, using the latter result, we show that, given a complex polynomial $f$, for each $m \geq 2$ we can compute an annulus containing the roots. In Section 5, we consider the application of the bounds on the modulus of roots within Weyl's algorithm. We conclude the paper in Section 6.

\section{THE BASIC FAMILY}

Assume that $f(z)$ is a complex polynomial of degree $n$. Define $D_{0}(z)=1$, and for each $m \geq 1$, let

$$
D_{m}(z)=\operatorname{det}\left(\begin{array}{ccccc}
f^{\prime}(z) & \frac{f^{\prime \prime}(z)}{2 !} & \ldots & \frac{f^{(m-1)}(x)}{(m-1) !} & \frac{f^{(m)}(z)}{(m) !} \\
f(z) & f^{\prime}(z) & \ddots & & \frac{f^{(m-1)}(z)}{(m-1) !} \\
0 & f(z) & \ddots & \ddots & \vdots \\
\vdots & \vdots & \ddots & \ddots & \frac{f^{\prime \prime}(z)}{2 !} \\
0 & 0 & \ldots & f(z) & f^{\prime}(z)
\end{array}\right)
$$


where det denotes determinant. Also, given any $m \geq 1$, for each $k \geq(m+1)$ define

$$
\widehat{D}_{m, k}(z)=\operatorname{det}\left(\begin{array}{ccccc}
\frac{f^{\prime \prime}(z)}{2 !} & \frac{f^{\prime \prime \prime}(z)}{3 !} & \ldots & \frac{f^{(m)}(z)}{(m) !} & \frac{f^{(k)}(z)}{k !} \\
f^{\prime}(z) & \frac{f^{\prime \prime}(z)}{2 !} & & \frac{f^{(m-1)}(z)}{(m-1) !} & \frac{f^{(k-1)}(z)}{(k-1) !} \\
f(z) & f^{\prime}(z) & \ddots & \vdots & \vdots \\
\vdots & \vdots & \ddots & \frac{f^{\prime \prime}(z)}{2 !} & \frac{f^{(k-m+2)}(z)}{(k-m+2) !} \\
0 & 0 & \ldots & f^{\prime}(z) & \frac{f^{(k-m+1)}(z)}{(k-m+1) !}
\end{array}\right) .
$$

Note that for $k \geq m+n, \widehat{D}_{m, k}(z)$ is identically zero. The following theorem is a consequence of results proved in Kalantari et al. [7] and Kalantari 9]. The main result of this paper makes use of the generalization of Theorem 2.1, stated for complex polynomials, to arbitrary analytic functions (Theorem 2.2).

Theorem 2.1. Assume that $f(z)$ is a complex polynomial of degree $n$. For each $m \geq 2$, define

$$
B_{m}(z) \equiv z-f(z) \frac{D_{m-2}(z)}{D_{m-1}(z)} .
$$

Let $\xi$ be a simple root of $f(z)$. Then

$$
B_{m}(z)=\xi+\sum_{k=m}^{m+n-2}(-1)^{m} \frac{\widehat{D}_{m-1, k}(z)}{D_{m-1}(z)}(\xi-z)^{k} .
$$

In particular, there exists $r>0$ such that given any $z_{0} \in N_{r}(\xi)=\{z:|z-\xi| \leq r\}$, the fixed-point iteration

$$
z_{i+1}=B_{m}\left(z_{i}\right), \quad i=1,2, \ldots,
$$

is well-defined and it converges to $\xi$ having order $m$. Specifically,

$$
\lim _{i \rightarrow \infty} \frac{\left(\xi-z_{i+1}\right)}{\left(\xi-z_{i}\right)^{m}}=(-1)^{m-1} \frac{\widehat{D}_{m-1, m}(\xi)}{D_{m-1}(\xi)}=(-1)^{m-1} \frac{\widehat{D}_{m-1, m}(\xi)}{p^{\prime}(\xi)^{m-1}} .
$$

The family of iteration functions $\left\{B_{m}(z)\right\}_{m=2}^{\infty}$ was derived in $[7$ and is called the basic family. Theorem 2.1 in particular gives the order of convergence of each $B_{m}$, and its asymptotic error constant. The first member, $B_{2}(z)$, is simply Newton's iteration function. The next member, $B_{3}(z)$, is credited to the astronomer Edmund Halley 3]. For the history, variations, and other interesting facts about Halley's method, see Scavo and Thoo [20], Traub [24, Ypma [26, and Kalantari [1]. In particular, Halley's method, which according to Traub [24] is the most rediscovered iteration function after Newton's, inspired the celebrated Taylor's theorem (see [26]).

Other individual members of the basic family have been rediscovered by several authors through different means. An earliest derivation of the basic family goes back to Schröder 21. (see its English translation by Stewart 21]). The basic family is sometimes also called König's family, see e.g. Vrscay and Gilbert 25]. What is often known in the literature as the Schröder and sometimes the Euler-Schröder family happens to be a second family of iteration functions; see, e.g., [4], [5], [22], [24]. A simple derivation of this latter family is given in Kalantari et al. 7] and [9].

Kalantari et al. [7] derive the basic family completely algebraically. This derivation reveals many interesting minimality and uniqueness properties of the basic 
family, in particular the important expansion given in (2.4). More generally, a nontrivial determinantal generalization of Taylor's theorem in Kalantari 9] gives a more general development of the basic family and its multipoint version. Each member $B_{m}$ of the basic family blossoms into $m$ iteration functions, $B_{m}^{(1)}=B_{m}, \ldots, B_{m}^{(m)}$, where $B_{m}^{(k)}$ is a $k$-point iteration function. The order of convergence of these iteration functions is derived in [8]. A simple development of the basic family for square and cube roots is given in [6]. For some other properties of the basic family see Kalantari and Gerlach [12, and Kalantari and Jin [16. An application of the basic family in deriving new formulas for approximation of $\pi$ is give in [13. Finally, 17] describes some visualization techniques using the basic family and its educational/artistic applications.

We will now proceed by proving a more general version of Theorem 2.1

Theorem 2.2. Let $f(z)$ be a complex-valued function analytic over the entire complex plane. For each $m \geq 2$, define $B_{m}(z)$ as in (2.3). Then if $\xi$ is a simple root of $f(z), B_{m}(z)$ satisfies the expansion

$$
B_{m}(z)=\xi+\sum_{k=m}^{\infty}(-1)^{m} \frac{\widehat{D}_{m-1, k}(z)}{D_{m-1}(z)}(\xi-z)^{k} .
$$

Sketch of Proof. Since $f(z)$ is analytic and $\xi$ a root from Taylor's theorem, we have

$$
0=f(\xi)=\sum_{k=0}^{\infty} \frac{f^{(k)}(z)}{k !}(\xi-z)^{k} .
$$

Adding and subtracting the quantity $z-f(z)=\xi-(\xi-z)-f(z)$ to both sides of $(2.8)$, we get

$$
B_{1}(z) \equiv z-f(z)=\xi+\left(f^{\prime}(z)-1\right)(\xi-z)+\sum_{k=2}^{\infty} \frac{f^{(k)}(z)}{k !}(\xi-z)^{k} .
$$

From (2.8) one can also easily obtain the following expansion for Newton's iteration, which coincides with (2.7) for $m=2$ :

$$
B_{2}(z) \equiv z-\frac{f(z)}{f^{\prime}(z)}=\xi+\sum_{k=2}^{\infty} \frac{f^{(k)}(z)}{k ! f^{\prime}(z)}(\xi-z)^{k} .
$$

Subtracting (2.10) from (2.9), we get

$$
\begin{aligned}
B_{1}(z)-B_{2}(z) & =-f(z)+\frac{f(z)}{f^{\prime}(z)} \\
& =\left(f^{\prime}(z)-1\right)(\xi-z)+\sum_{k=2}^{\infty} \frac{\left(f^{\prime}(z)-1\right) f^{(k)}(z)}{k ! f^{\prime}(z)}(\xi-z)^{k} .
\end{aligned}
$$

Multiplying (2.8) by $-\left(f^{\prime}(z)-1\right)(\xi-z)$, and (2.11) by $f(z)$ and then adding the results, we get

$$
f(z)\left(B_{1}(z)-B_{2}(z)\right)=f(z)^{2} \frac{\left(1-f^{\prime}(z)\right)}{f^{\prime}(z)}=\sum_{k=2}^{\infty} u_{k}(z)(\xi-z)^{k},
$$

where for $k \geq 2$

$$
u_{k}(z)=\left(f^{\prime}(z)-1\right)\left(\frac{f(z) f^{(k)}(z)}{k ! f^{\prime}(z)}-\frac{f^{(k-1)}(z)}{(k-1) !}\right) .
$$


Multiplying (2.12) by

$$
-\frac{f^{\prime \prime}(z)}{2 f^{\prime}(z)} \frac{1}{u_{2}(z)}=-\frac{f^{\prime \prime}(z)}{\left(1-f^{\prime}(z)\right)\left(2 f^{\prime}(z)^{2}-f(z) f^{\prime \prime}(z)\right)}
$$

and adding the results to (2.10) and simplifying, we get a new iteration function:

$$
\begin{aligned}
B_{3}(z) & \equiv z-\frac{f(z)}{f^{\prime}(z)}-\frac{f(z)^{2} f^{\prime \prime}(z)}{f^{\prime}(z)\left(2 f^{\prime}(z)^{2}-f(z) f^{\prime \prime}(z)\right)} \\
& =z-f(z) \frac{f^{\prime}(z)}{f^{\prime}(z)^{2}-f(z) f^{\prime \prime}(z) / 2}=z-f(z) \frac{D_{1}(z)}{D_{2}(z)} .
\end{aligned}
$$

The corresponding expansion for $B_{3}(z)$ becomes

$$
\begin{aligned}
B_{3}(z) & =\xi-\sum_{k=3}^{\infty}\left(\frac{f^{(k)}(z)}{k ! f^{\prime}(z)}-\frac{f^{\prime \prime}(z)}{2 f^{\prime}(z)} \frac{u_{k}(z)}{u_{2}(z)}\right)(\xi-z)^{k} \\
& =\xi-\sum_{k=3}^{\infty}\left[\left(\frac{f^{(k)}(z) f^{\prime}(z)}{k !}-\frac{f^{\prime \prime}(z) f^{(k-1)}(z)}{2(k-1) !}\right)\right. \\
& \left.\quad \div\left(f^{\prime}(z)^{2}-\frac{1}{2} f(z) f^{\prime \prime}(z)\right)\right](\xi-z)^{k} \\
= & \xi-\sum_{k=3}^{\infty} \frac{\widehat{D}_{2, k}(z)}{D_{2}(z)}(\xi-z)^{k} .
\end{aligned}
$$

This proves the theorem for $m=3$ since it coincides with (2.7).

In general we can recursively obtain $B_{m+1}(z)$ and its corresponding expansion, given $B_{m}(z), B_{m-1}(z)$ and their expansions in the same way that we obtained $B_{3}(z)$ from $B_{2}(z)$ and $B_{1}(z)$. The fact that this process is well-defined, together with the claimed expansion, has already been proved for polynomials in Kalantari et al. [7] and Kalantari [9. We thus do not give a complete proof, which would just replace polynomials with analytic functions.

\section{Application OF the BASIC FAMILY IN DERIVING SEPARATION THEOREMS}

In this section we will make use of the basic family and Theorem 2.2 to derive a family of lower bounds on the distance from a given zero of $f(z)$ to its nearest distinct zero. Define

$$
\gamma_{m}(z)=\sup \left\{\left|\frac{\widehat{D}_{m-1, k}(z)}{D_{m-1}(z)}\right|^{1 /(k-1)}: k \geq m\right\} .
$$

The following is a key result.

Proposition 3.1. Assume $\xi$ is a simple root of $f(z)$ and let $u=|z-\xi| \gamma_{m}(z)$. If $u<1$, then

$$
\left|B_{m}(z)-\xi\right| \leq \frac{u^{m-1}}{1-u}|\xi-z|=\frac{\gamma_{m}^{m-1}(z)|\xi-z|^{m}}{1-u} .
$$

Proof. From the definition of $\gamma_{m}(z)$ and from the expansion formula (2.4) for $B_{m}(z)$ in Theorem 2.2 we have

$$
\left|B_{m}(z)-\xi\right| \leq \sum_{k=m}^{\infty} \gamma_{m}(z)^{k-1}|\xi-z|^{k}=|\xi-z| \sum_{k=m}^{\infty} u^{k-1} .
$$


Since $u<1$,

$$
\sum_{k=m}^{\infty} u^{k-1}=u^{m-1} \sum_{k=0}^{\infty} u^{k}=\frac{u^{m-1}}{(1-u)}
$$

Thus we get

$$
\left|B_{m}(z)-\xi\right| \leq \frac{u^{m-1}}{(1-u)}|\xi-z|=\frac{\gamma_{m}^{m-1}(z)|\xi-z|^{m}}{(1-u)} .
$$

Hence the proof.

We now have

Theorem 3.2. If $\xi, \xi^{\prime}$ are zeros of $f, \xi$ a simple zero, then they are separated by a distance

$$
\left|\xi-\xi^{\prime}\right| \geq \frac{r_{m}}{\gamma_{m}(\xi)}
$$

where $r_{m} \in[1 / 2,1)$ is the unique positive root of the polynomial $t^{m-1}+t-1$. In particular, if $m=2$, then $r_{m}=1 / 2 ; \gamma_{m}(\xi)$ coincides with $\gamma(\xi)$ (see (1.2)), and we get

$$
\left|\xi-\xi^{\prime}\right| \geq \frac{1}{2 \gamma(\xi)}
$$

Proof. The polynomial $q(t)=t^{m-1}+t-1$ has a unique positive root $r_{m}<1$. The existence, uniqueness, and bound follow from the inequalities $q(0)<0, q(1)>1$, and the fact that since $q^{\prime}(t)>0, q(t)$ is monotonically increasing for positive $t$.

Since $r_{m}<1$, if $u=\gamma_{m}(\xi)\left|\xi^{\prime}-\xi\right| \geq 1$, then the lower bound in the statement is already satisfied. So assume that $u=\gamma_{m}(\xi)\left|\xi^{\prime}-\xi\right|<1$. Note that since $\xi^{\prime}$ is a root of $f$, we have $D_{m}\left(\xi^{\prime}\right)=f^{\prime}\left(\xi^{\prime}\right)^{m}$. Thus, whether or not $\xi^{\prime}$ is a simple root of $f$, it is a fixed-point of $B_{m}$ since we have

$$
B_{m}\left(\xi^{\prime}\right)=\xi^{\prime}-f\left(\xi^{\prime}\right) \frac{f^{\prime}\left(\xi^{\prime}\right)^{m-2}}{f^{\prime}\left(\xi^{\prime}\right)^{m-1}}=\xi^{\prime}-\frac{f\left(\xi^{\prime}\right)}{f^{\prime}\left(\xi^{\prime}\right)}=\xi^{\prime} .
$$

Then, substituting $\xi^{\prime}$ for $z$ in Proposition 3.1 since $\xi^{\prime}$ is a fixed point of $B_{m}(z)$ we get

$$
\left|B_{m}\left(\xi^{\prime}\right)-\xi\right|=\left|\xi^{\prime}-\xi\right| \leq \frac{u^{m-1}}{(1-u)}\left|\xi^{\prime}-\xi\right| .
$$

Dividing the above by $\left|\xi^{\prime}-\xi\right|$ yields $u^{m-1} \geq 1-u$. Equivalently, $q(u)=u^{m-1}+$ $u-1 \geq 0$. Thus we must have

$$
u=\gamma_{m}(\xi)\left|\xi-\xi^{\prime}\right| \geq r_{m} .
$$

Equivalently,

$$
\left|\xi-\xi^{\prime}\right| \geq \frac{r_{m}}{\gamma_{m}(\xi)}
$$

In particular, if $m=2$, then $r_{2}=1 / 2$, and we get

$$
\left|\xi-\xi^{\prime}\right| \geq \frac{1}{2 \gamma_{2}(\xi)}=\frac{1}{2 \gamma(\xi)} .
$$

Hence the proof.

Remark 1. For polynomials of degree $n$ and small values of $m$, given $\xi$, the quantity $\gamma_{m}(\xi)$ is computable within the same complexity as the normalized derivatives of $f(z)$, which can be done in $O(n \log n)$ (see Pan [19]). 
Remark 2. For $m=2$ our lower bound is more than twice as good as Smale's lower bound (1.3) as well as the Blum et al. bound (1.4). It should be mentioned that these bounds are auxiliary results obtained within the context of the analysis of Smale's one-point theory on Newton's method (see Smale [23, or Blum et al. [1]). That analysis required a more conservative expansion formula for Newton's iteration function than the expansion given as (2.10) here. In [18 we will make use of Smale's analysis and some of the results proved in this paper to give a generalization of Smale's one-point theory for the entire basic family.

Example 1. We now examine the quality of our lower bound for a simple example. We will consider $f(z)=z^{2}-1$. More generally, we could consider $z^{2}-\xi^{2}$ (or in fact an arbitrary quadratic polynomial), but the results will be analogous to the case of $z^{2}-1$. Then $\xi=1, \xi^{\prime}=-1$. Then $f^{\prime}(\xi)=2, f^{\prime \prime}(\xi) / 2 !=1$, and $f^{\prime \prime \prime}(\xi)=0$. For any $m \geq 2$ we have $\widehat{D}_{m-1, k}(\xi)=0$, whenever $k>m$. Thus (3.1) implies

$$
\gamma_{m}(\xi)=\left(\frac{\widehat{D}_{m-1, m}(\xi)}{D_{m-1}(\xi)}\right)^{1 /(m-1)} .
$$

But it is easy to see that $D_{m-1}(\xi)=f^{\prime}(\xi)^{m-1}=2^{m-1}$, and $\widehat{D}_{m-1, m}(\xi)=1$. Thus, $\gamma_{m}(\xi)=1 / 2$, and according to Theorem 3.2

$$
\left|\xi-\xi^{\prime}\right| \geq 2 r_{m}
$$

When $m=2$ we have $r_{m}=1 / 2$, and thus the lower bound is only 1 . When $m=3$, $r_{m}$ is the positive root of $u^{2}+u-1$, i.e., the lower bound is $\sqrt{5}-1$. As $m$ goes to infinity, $r_{m}$ converges to 1 and hence the lower bounds converge to the actual gap, namely 2 .

Remark 3. Estimation of the quantity $\gamma_{m}(\xi)$ (see (3.1) ) for $m$ large and for a general polynomial or analytic function can be cumbersome. For this reason we may consider an estimate of this quantity which can be computed efficiently. If for each $k$ we have an upper bound $M_{k}$ on the modulus of column $k$ of the matrix corresponding to $\widehat{D}_{m-1, k}(\xi)$, then from Hadamard's bound on determinants we have

$$
\left|\widehat{D}_{m-1, k}(\xi)\right| \leq \prod_{k=1}^{m-1} M_{k}
$$

Also $D_{m-1}(\xi)=f^{\prime}(\xi)^{m-1}$. Since $M_{k}$ can easily be calculated, we see that an efficiently computable upper bound on $\gamma_{m}(\xi)$ may be available.

\section{Estimate to A NEAREST ZERO AND UPPER AND LOWER BOUNDS ON ZEROS}

The previous section gives lower bounds on the distance between a given root of $f(z)$ and its nearest root. In this section we show that the above results can be used to estimate the distance to the nearest zero of $f(z)$ for an arbitrary point in the complex plane. Before doing so, let us recall the quantities $\gamma_{m}(z), D_{m-1}(z)$, $\widehat{D}_{m-1, k}(z)$ defined in (3.1), (2.1),$(2.2)$, respectively. We will first represent these as two-variable functions

$$
\gamma_{m}(z, f), D_{m-1}(z, f), \widehat{D}_{m-1, k}(z, f),
$$

to indicate that they are defined with respect to a given $z$ and a given function $f$.

We next prove 
Theorem 4.1. Let $z_{0}$ be a given complex number different than a root of $f(z)$. Let $F(z)=f(z)\left(z-z_{0}\right)$. For a given $m \geq 2$, define

$$
\gamma_{m}(z, F)=\sup \left\{\left|\frac{\widehat{D}_{m-1, k}(z, F)}{D_{m-1}(z, F)}\right|^{1 /(k-1)}: k \geq m\right\} .
$$

Then, if $\xi$ is any root of $f(z)$, we have

$$
\left|z_{0}-\xi\right| \geq \frac{r_{m}}{\gamma_{m}\left(z_{0}, F\right)}
$$

where $r_{m} \in[1 / 2,1)$ is the unique positive root of the polynomial $t^{m-1}+t-1$. In particular, for $m=2, r_{m}=1 / 2$, we have

$$
\gamma_{2}\left(z_{0}, F\right)=\sup \left\{\left|\frac{f^{(k)}\left(z_{0}\right)}{f\left(z_{0}\right) k !}\right|^{1 / k}, k \geq 1\right\},
$$

and

$$
\left|z_{0}-\xi\right| \geq \frac{1}{2 \gamma_{2}\left(z_{0}, F\right)}
$$

and for $m=3, r_{m}=(\sqrt{5}-1) / 2$, we have

$$
\gamma_{3}\left(z_{0}, F\right)=\max \left\{\left|\frac{1}{f\left(z_{0}\right)^{2}} \operatorname{det}\left(\begin{array}{cc}
f^{\prime}\left(z_{0}\right) & \frac{f^{(k)}\left(z_{0}\right)}{\left(z_{0}\right)} \\
f(k-1) !
\end{array}\right)\right|^{1 / k}: k \geq 2\right\},
$$

and

$$
\left|z_{0}-\xi\right| \geq \frac{\sqrt{5}-1}{2 \gamma_{3}\left(z_{0}, F\right)} .
$$

Proof. The first assertion is merely the application of Theorem 3.2 to $F(z)$ at its root $z_{0}$. To prove the formula for $\gamma_{2}\left(z_{0}, F\right)$ requires us to verify that

$$
F^{(k)}\left(z_{0}\right)=k f^{(k-1)}\left(z_{0}\right) .
$$

But this can be shown easily. This gives

$$
\gamma_{2}\left(z_{0}, F\right)=\sup \left\{\left|\frac{F^{(k)}\left(z_{0}\right)}{F^{\prime}\left(z_{0}\right) k !}\right|^{1 /(k-1)}, k \geq 2\right\}=\sup \left\{\left|\frac{f^{(k-1)}\left(z_{0}\right)}{f\left(z_{0}\right)(k-1) !}\right|^{1 /(k-1)}, k \geq 2\right\} .
$$

Now, changing $k-1$ to $k$, we obtain the claimed result for $\gamma_{2}\left(z_{0}, F\right)$, and hence the lower bound on the gap $\left|z_{0}-\xi\right|$.

For $m=3, r_{m}$ is the positive root $t^{2}+t+1$. This is as claimed. Next we need to compute $\gamma_{3}\left(z_{0}, F\right)$. From (4.2) we have

$$
D_{2}\left(z_{0}, F\right)=\operatorname{det}\left(\begin{array}{cc}
F^{\prime}\left(z_{0}\right) & \frac{F^{\prime \prime}\left(z_{0}\right)}{2 !} \\
F\left(z_{0}\right) & F^{\prime}\left(z_{0}\right)
\end{array}\right)=\operatorname{det}\left(\begin{array}{cc}
f\left(z_{0}\right) & f^{\prime}\left(z_{0}\right) \\
0 & f\left(z_{0}\right)
\end{array}\right)=f\left(z_{0}\right)^{2} .
$$

For each $k \geq 3$ from (4.2) we have

$$
\widehat{D}_{m-1, k}\left(z_{0}, F\right)=\operatorname{det}\left(\begin{array}{cc}
\frac{F^{\prime \prime}\left(z_{0}\right)}{2 !} & \frac{F^{(k)}\left(z_{0}\right)}{k !} \\
F^{\prime}\left(z_{0}\right) & \frac{F^{(k-1)}\left(z_{0}\right)}{(k-1) !}
\end{array}\right)=\operatorname{det}\left(\begin{array}{cc}
f^{\prime}\left(z_{0}\right) & \frac{f^{(k-1)}\left(z_{0}\right)}{(k-1) !} \\
f\left(z_{0}\right) & \frac{f^{(k-2)}\left(z_{0}\right)}{(k-2) !}
\end{array}\right) .
$$

Next, changing $k-1$ to $k$, we get the claimed quantity for $\gamma_{3}\left(z_{0}, F\right)$. 
We will now state two corollaries of the above, giving upper and lower bounds on the modulus of polynomial roots. We will refer to the first as a second-order bound since it is based on $m=2$, and the next as third-order bound since it is based on $m=3$. More generally, for any natural number $m \geq 2$ we can state an $m t h$-order bound.

Corollary 4.2 (Second-order lower bound). Assume that $f(z)=a_{n} z^{n}+a_{n-1} z^{n-1}+$ $\cdots+a_{0}, a_{n} a_{0} \neq 0$. Then the modulus of each root of $f$ is bounded below by the quantity

$$
L_{2} \equiv \frac{1}{2}\left(\max \left\{\left|\frac{a_{k}}{a_{0}}\right|^{1 / k}: k=1, \ldots, n\right\}\right)^{-1} .
$$

Proof. Let $m=2$, set $z_{0}=0$ and apply Theorem 4.1, using that $f(0)=a_{0}$, $f^{(k)}(0) / k !=a_{k}$.

Corollary 4.3 (Third-order lower bound). Assume that $f(z)=a_{n} z^{n}+a_{n-1} z^{n-1}+$ $\cdots+a_{0}, a_{n} a_{0} \neq 0$. Then the modulus of each root of $f$ is bounded below by the quantity

$$
L_{3} \equiv \frac{\sqrt{5}-1}{2}\left(\max \left\{\left(\frac{\left|a_{1} a_{k-1}-a_{0} a_{k}\right|}{\left|a_{0}^{2}\right|}\right)^{1 / k}: k=2, \ldots, n+1\right\}\right)^{-1},
$$

where $a_{n+1} \equiv 0$.

Proof. Let $m=3$, set $z_{0}=0$ and apply Theorem 4.1 to compute $\gamma_{3}\left(z_{0}, F\right)$. First observe that

$$
\operatorname{det}\left(\begin{array}{cc}
f^{\prime}(0) & \frac{f^{(k)}(0)}{f^{(k-1)}(0)} \\
f(0) & \frac{f^{(k-1) !}}{(k-1)}
\end{array}\right)=\operatorname{det}\left(\begin{array}{cc}
a_{1} & a_{k} \\
a_{0} & a_{k-1}
\end{array}\right)=a_{1} a_{k-1}-a_{0} a_{k} .
$$

The index $k$ ranges from 2 to $n+1$, and since $f^{(n+1)}(0)=0$, we can set $a_{n+1}=0$. Also $f(0)^{2}=a_{0}^{2}$. Hence we have

$$
\gamma_{3}\left(z_{0}, F\right)=\max \left\{\left(\frac{\left|a_{1} a_{k-1}-a_{0} a_{k}\right|}{\left|a_{0}^{2}\right|}\right)^{1 / k}: k=2, \ldots, n+1\right\} .
$$

From this and Theorem 4.1 we get the desired lower bound on $\left|z_{0}-\xi\right|$.

Applying the above corollaries to the polynomial

$$
g(z)=z^{n} f\left(\frac{1}{z}\right)=a_{0} z^{n}+a_{1} z^{n-1}+\cdots+a_{n-1} z+a_{n},
$$

we obtain second and third order lower bounds on the roots of $g(z)$. But since the roots of $g(z)$ are the reciprocal of the roots of $f(z)$, we obtain the following second and third order upper bounds to the modulus of the roots of $f(z)$.

Corollary 4.4 (Second-order upper bound). Assume that $f(z)=a_{n} z^{n}+a_{n-1} z^{n-1}+$ $\cdots+a_{0}, a_{n} a_{0} \neq 0$. Then the modulus of each root of $f$ is bounded above by the quantity

$$
U_{2} \equiv 2 \max \left\{\left|\frac{a_{n-k}}{a_{n}}\right|^{1 / k}: k=1, \ldots, n\right\}
$$


Corollary 4.5 (Third-order upper bound). Assume that $f(z)=a_{n} z^{n}+a_{n-1} z^{n-1}+$ $\cdots+a_{0}, a_{n} a_{0} \neq 0$. Then the modulus of each root of $f$ is bounded above by the quantity

$$
U_{3}=\equiv \frac{\sqrt{5}+1}{2} \max \left\{\left(\frac{\left|a_{n-1} a_{n-k+1}-a_{n} a_{n-k}\right|}{\left|a_{n}^{2}\right|}\right)^{1 / k}: k=2, \ldots, n+1\right\},
$$

where $a_{-1} \equiv 0$.

Remark 4. The upper bound given in Corollary 4.4 was derived through different means in Henrici [4] (Corollary 6.4k, page 457). Also a more relaxed version of it is given in Blum et al. [1] (Lemma 2, page 170).

Example 2. We consider a simple example to indicate the utility of the last four corollaries. Let $f(z)=z^{n}-1$, where the roots are roots of unity. From the above four corollaries we deduce the following second- and third-order upper and lower bounds:

$$
\begin{gathered}
\left\{z: L_{2} \leq|z| \leq U_{2}\right\}=\left\{z: \frac{1}{2} \leq|z| \leq 2\right\}, \\
\left\{z: L_{3} \leq|z| \leq U_{3}\right\}=\left\{z: \frac{\sqrt{5}-1}{2} \leq|z| \leq \frac{\sqrt{5}+1}{2}\right\} .
\end{gathered}
$$

We see that the annulus reduces in size as we go from $m=2$ to $m=3$. It is tempting to conclude that as $m$ increases the annulus converges to the circle of radius one, i.e., the tightest possible annulus containing the roots. We will establish this elsewhere by proving that for each $m \geq 2$ the roots lie in the annulus

$$
\left\{z: L_{m} \leq|z| \leq U_{m}\right\}=\left\{z: r_{m} \leq|z| \leq r_{m}^{-1}\right\} .
$$

\section{Applications}

Here we discuss some applications of the above bounds. Clearly it is always desirable to have lower and upper bounds on the roots of a given analytic function. But one direct application is in Weyl's algorithm for computing all roots of a given complex polynomial (see Henrici [4], Pan [19], Weyl [27]). The algorithm can be viewed as a two-dimensional version of the bisection algorithm. It begins with an initial suspect square containing all the roots. Given a suspect square, we partition it into four congruent subsquares. At the center of each of the four subsquares we perform a proximity test, i.e., we estimate the distance from the center to the nearest zero. If the proximity test guarantees that the distance exceeds half of the length of the diagonal of the square, then the square cannot contain any zeros and it is discarded. The remaining squares are called suspect, and each of them will recursively be partitioned into four congruent subsquares and the process repeated. We see that our lower bounds can be used as alternative proximity tests to the existing ones, possibly much more effective since we have exhibited infinitely many lower bounds. Moreover, our upper bounds can be used to obtain a tight initial suspect square.

\section{Concluding Remarks}

In this paper we have made use of the basic family to arrive at infinitely many new lower bounds on the distance between a zero of an analytic function or a complex polynomial and its nearest distinct zero. We then showed how to obtain from these lower bounds an estimate of the distance to the nearest zero for an arbitrary point 
in the complex plane. Moreover, we showed that for each natural number $m \geq 2$ we can obtain an $m$ th-order upper and lower bound on the modulus of the roots of complex polynomials. These bounds in particular suggest a new proximity test in Weyl's algorithm for the computation of all roots of complex polynomials.

From the practical point of view, for small values of $m$ the bounds are efficiently computable. We will report on their effectiveness in Weyl's algorithm in the future. From the theoretical point of view our bounds on zeros provide alternatives to existing bounds on the modulus of zeros. A detailed comparison with some existing bounds is worthy of a separate note. Our results also suggest new research problems. For instance, is it the case that for every complex polynomial the upper and lower bounds converge to the tightest annulus containing the roots? What is the efficiency of computing the upper and lower bounds as a function of $n$, the degree of the polynomial and the parameter $m$ ? Finally, in [18] we will make use of some of the results proved in this paper to give a generalization of Smale's one-point theory on Newton's method to the entire basic family.

\section{ACKNOWLEDGMENT}

I would like to thank an anonymous referee.

\section{REFERENCES}

[1] L. Blum, F. Cucker, M. Shub, S. Smale, Complexity and Real Computation, Springer-Verlag, New York, 1998. MR 99a:68070

[2] J.-P. Dediue, Estimation for the separation number of a polynomial system, J. Symbolic Computation 24 (1997), 683-693. MR 99b:65065

[3] E. Halley, A new, exact, and easy method of finding roots of any equations generally, and that without any previous reduction, Philos. Trans. Roy. Soc. London, 18 (1694), 136-145.

[4] P. Henrici, Applied and Computational Complex Analysis, Vol. I, Wiley, New York, 1974. MR 51:8378

[5] A.S. Householder, The Numerical Treatment of a Single Nonlinear Equation, McGraw-Hill, New York, 1970. MR 52:9593

[6] B. Kalantari, and I. Kalantari, High order iterative methods for approximating square roots, BIT 36 (1996), 395-399. MR 97k:65039

[7] B. Kalantari, I. Kalantari, and R. Zaare-Nahandi, A basic family of iteration functions for polynomial root finding and its characterizations, J. of Comp. and Appl. Math., 80 (1997), 209-226. MR 98d:65066

[8] B. Kalantari, On the order of convergence of a determinantal family of root-finding methods, BIT 39 (1999), 96-109. MR 2000b:65099

[9] B. Kalantari, Generalization of Taylor's theorem and Newton's method via a new family of determinantal interpolation formulas and its applications, J. of Comp. and Appl. Math., 126 (2000), 287-318. MR 2001m:41035

[10] B. Kalantari, Approximation of polynomial root using a single input and the corresponding derivative values, Technical Report DCS-TR 369, Department of Computer Science, Rutgers University, New Brunswick, New Jersey, 1998.

[11] B. Kalantari, Halley's method is the first member of an infinite family of cubic order rootfinding methods, Technical Report DCS-TR 370, Department of Computer Science, Rutgers University, New Brunswick, New Jersey, 1998.

[12] B. Kalantari and J. Gerlach, Newton's method and generation of a determinantal family of iteration functions, J. of Comp. and Appl. Math., 116 (2000), 195-200. MR 2000k:65098

[13] B. Kalantari, New formulas for approximation of $\pi$ and other transcendental numbers, $N u$ merical Algorithms 24 (2000), 59-81. MR 2001h:11087

[14] B. Kalantari, On homogeneous linear recurrence relations and approximation of zeros of complex polynomials, in Unusual Applications in Number Theory (M.B. Nathanson, Ed.), DIMACS Series in Discrete Mathematics and Theoretical Computer Science, vol. 64, 2000, pp. 125-143. 
[15] B. Kalantari and S. Park, A computational comparison of the first nine members of a determinantal family of root-finding methods, J. of Comp. and Appl. Math., 130 (2001), 197-204. MR 2002b:65079

[16] B. Kalantari and Y. Jin, On extraneous fixed-points of the basic family of iteration functions, BIT, 43 (2003), 453-458.

[17] B. Kalantari, Polynomiography: A new intersection between mathematics and art, Technical Report DCS-TR 506, Department of Computer Science, Rutgers University, New Brunswick, New Jersey, 2002 (www.polynomiography.com)

[18] B. Kalantari, A generalization of Smale's one-point theory for the basic family, Department of Computer Science, Rutgers University, New Brunswick, New Jersey, forthcoming.

[19] V. Y. Pan, Solving a polynomial equation: some history and recent progress, SIAM Review, 39 (1997), 187-220. MR 99b:65066

[20] T. R. Scavo and J. B. Thoo, On the geometry of Halley's method, Amer. Math. Monthly, 102 (1995), 417-426. MR 96f:01019

[21] E. Schröder, On infinitely many algorithms for solving equations (German), Math. Ann. 2 (1870) 317-365.(English translation by G.W. Stewart, TR-92-121, Institute for Advanced Computer Studies, University of Maryland, College Park, MD, 1992.)

[22] M. Shub and S. Smale, Computational complexity: On the geometry of polynomials and a theory of cost, Part I, Ann. Sci. Ecole Norm. Sup., 18 (1985), 107-142. MR 87m:68043

[23] S. Smale, Newton's method estimates from data at one point, in The Merging of Disciplines: New Directions in Pure, Applied, and Computational Mathematics, R. E. Ewing, K.I. Gross, C. F. Martin, eds., Springer-Verlag, New York, pp. 185-196, 1986. MR 88e:65076

[24] J. F. Traub, Iterative Methods for the Solution of Equations, Englewood Cliffs, NJ, Prentice Hall, 1964. MR 29:6607

[25] E. R. Vrscay and W. J. Gilbert, Extraneous Fixed Points, Basin Boundaries and Chaotic Dynamics for Schröder and König Iteration Functions, Numer. Math. 52, 1-16 (1988). MR 89b:30026

[26] T. J. Ypma, Historical development of Newton-Raphson method, SIAM Review, 37 (1995), 531-551. MR 97b:01003

[27] H. Weyl, Randbemerkungen zu Hauptproblemen der Mathematik. II. Fundamentalsatz der Algebra und Grundlagen der Mathematik, Math. Z. 20 (1924), 142-150.

Department of Computer Science, Rutgers University, New Brunswick, New Jersey 08903

E-mail address: kalantar@cs.rutgers.edu 